\title{
Efectos de disoluciones nutritivas de nitrógeno, fósforo y potasio (N-P-K) sobre el crecimiento de plántulas de Juglans Neotropica Diels
}

Effects of nutritive dissolutions of nitrogen, phosphorus and potassium (N-P-K) on the growth of plantules of Juglans Neotropica Diels

Verónica Susana Guambo Tapia. ${ }^{1}$, Darwin Augusto Valenzuela Erazo. ${ }^{2}$, Angelica María Saeteros Hernández. ${ }^{3}$ \& Vilma Fernanda Noboa Silva. ${ }^{4}$

\begin{abstract}
DOI: https://doi.org/10.33262/cienciadigital.v4i1.1097

The production of quality seedlings constitutes a strategy to ensure survival and development and thus face the natural changes and those caused by human activity that endanger forest species. This investigation aims to characterize the morphological response of the provenances of $J$. neotropics to the nutritive solutions of NPK. This is an experimental, descriptive and longitudinal study that established a randomized complete block design (DBCA) with 262 observations, growth development was evaluated in 65 days from 30 days after sowing; measures of height increase and the increase of the stem diameter of the plants were taken. Descriptive statistical analysis, dispersion and central tendency, analysis of variance, and a cluster were executed to determine clusters of growth patterns with respect to provenances. Significant differences were found in the response of the plants and where T6 obtained the best results in height and diameter. It was concluded that the best treatment is $\mathrm{T} 6$ and with respect to provenances $\mathrm{P} 4$ has the best growth values, these results differ from the control and other authors. The cluster procedure by linking
\end{abstract}

\footnotetext{
${ }^{1}$ Gad Municipal Riobamba, Chimborazo, Riobamba, guambove@gadmriobamba.gob.ec

${ }^{2}$ Escuela Superior Politécnica de Chimborazo, Riobamba, darwin.valenzuela@espoch.edu.ec

${ }^{3}$ Gad Municipal Riobamba, Chimborazo, Riobamba, saeterosa@gadmriobamba.gob.ec

${ }^{4}$ Escuela Superior Politécnica de Chimborazo, Riobamba, vilma.noboa@espoch.edu.ec
} 
Ward through the Euclidean distance grouped growth trends into three groups without finding incongruity factors. Pearson's Chi-square test shows that the three groups found can be associated with the morphological response of $\mathrm{J}$. neotropics from different sources to the treatments. It is recommended to study the genetics of these varieties of J. neotropics to show if the origin has generated sub varieties with different growth profiles and nutritional needs.

Key words. J. Neotropics, Nourishing Solutions, Walnut Growth.

\section{Resumen}

La productividad de plantines de calidad constituye una estrategia para asegurar la supervivencia y el desarrollo y de esta manera enfrentar los cambios naturales y los ocasionados por la actividad humana que ponen en peligro diversas especies forestales. El objetivo de esta investigación es describir morfológicamente las respuestas de variedades de J. neotropical a las disoluciones nutritivas de N P K. Esta investigación es experimental, descriptiva y con enfoque longitudinal que estableció un diseño en bloques completos al azar (DBCA) con 262 observaciones, se evaluó el desarrollo del crecimiento en 65 días desde los 30 días después de haberse sembrado; se tomaron medidas de incremento de altura y el incremento del diámetro del tallo de las plantas. Se realizaron análisis estadísticos descriptivos, de dispersión y tendencia central, análisis de varianza, y se ejecutó un clúster para determinar agrupaciones de patrones de crecimiento respecto de las procedencias. Se encontraron diferencias significativas a la respuesta de las plantas y donde T6 obtuvo los mejores resultados en altura y en diámetro. Se concluyó que el mejor tratamiento es T6 y respecto de las procedencias P4 tiene los mejores valores de crecimiento, estos resultados difieren del testigo y de otros autores. El procedimiento clúster por medio de la vinculación de Ward a través de la distancia Euclidiana agrupó las tendencias de crecimiento en tres grupos sin encontrarse factores de incongruencia. La prueba de Chi-cuadrado de Pearson demuestra que los tres grupos encontrados pueden asociarse con la respuesta morfológica de J. neotropica de distintas procedencias a los tratamientos. Se recomienda estudiar la genética de estas variedades de J. neotropica para 
demostrar si la procedencia ha generado sub variedades con perfiles de crecimiento y necesidades nutricionales diferentes.

Palabras claves. J. Neotropica, Disoluciones Nutritivas, Crecimiento del Nogal.

\section{Introducción}

Jara (2015 citado en Guambo, 2018) considera deforestación al fenómeno por el cual se va destruyendo toda la superficie forestal de un bosque mediante acciones humanas producidas directamente sobre la naturaleza (Jara, 2015). La deforestación en Ecuador es un problema ambiental significativo por ejemplo desde 2008 al 2012 la deforestación se registró en 65.880 ha/año para una tasa de $-0,54 \%$ (Ramos \& Arias, 2016).

La deforestación es el fenómeno por el cual la cubierta forestal de un bosque es eliminada totalmente por medio de acciones producidas directamente por el hombre, sistemáticamente (Guambo, 2018). La deforestación es causada por la mala acción humana sobre la naturaleza, principalmente la tala de bosques realizada por industrias madereras sin regulaciones, la eliminación de árboles dentro de zonas de vocación forestal con el objetivo de preparar terrenos con fines agropecuarios, a los que se suman los incendios forestales (Paiz Girón, 2006).

Salto et al. (2016 citado en Guambo, 2018) resaltan la importancia de la productividad de plantines de calidad asegurando los indicadores como supervivencia y adecuado desarrollo. Basave et al. (2014) demostraron que, al utilizar plantas competitivas producidas en vivero, se logra altas tasas de supervivencia y crecimiento.

En la obtención de plantas vigorosas, es recomendable aplicar prácticas culturales adecuadas como la fertilización en vivero, así también la utilización de germoplasma de calidad. Por lo antes expuesto es sumamente importante evaluar la fertilización de la especie de J. neotropica con disoluciones nutritivas para la producción de plantines en vivero, con la finalidad de determinar soluciones nutritivas ajustadas a los requerimientos de esta especie que permita obtener plantas de calidad que aseguren la supervivencia, crecimiento y aclimatación en el campo.

La especie J. neotropica más conocida como nogal o tocte es utilizada en programas de forestación y reforestación con fines de conservación y producción (Peña et al. 2014). Según Toro y Roldán (2018) J. neotropica es originaria de América del Sur y se encuentra entre $1800 \mathrm{~m}$ y $2800 \mathrm{~m}$ de altitud, en bosques secos y húmedos montanos bajos. 
En Ecuador pese a tener las condiciones favorables en la región Andina para su crecimiento y desarrollo, no existe plantaciones como tal, ya que esta planta se puede encontrar de manera esporádica formando parte de los huertos familiares, en asociaciones con otros frutales, como cercas vivas, entre otras especies (Guambo, 2018).

Se han encontrado pequeñas plantaciones de nogal, en la provincia de Loja en la comunidad de El Tundo, pequeñas huertas experimentales en la ESPOCH- Riobamba, y en el norte, se puede encontrar en la hacienda San Antonio provincia de Imbabura (Azas, 2016 citado en Guambo, 2018).

Esta investigación se enfoca en el efecto de distintas alternativas de disoluciones nutritivas a distintas concentraciones con base en NPK para la fertilización en el desarrollo y crecimiento de plantines de $\mathrm{J}$ neotropica producidos bajo invernadero, que permitan generar plantas vigorosas que aseguren su establecimiento en campo que sean una alternativa para el control de la deforestación en condiciones similares a las del estudio que permitan aprovechar las bondades del nogal negro (Guambo, 2018).

El nogal (J. neotropica) es una especie de gran potencial para proyectos de conservación y agro productivos en la eco-región Andina. No obstante, su baja capacidad germinativa limita su propagación para reforestación (Cabascango, 2011),

Entre las especies andinas, J. neotropica, es de particular importancia para las comunidades locales, ya que además de proporcionar cobertura vegetal, esta especie es aprovechada también por sus propiedades medicinales probablemente se requieren periodos de estratificación mayores, entre 90 y 120 días. Debido a su baja germinación natural, los métodos de propagación asistida in vitro representan una alternativa para el abastecimiento de grandes números de plantas para la propagación de estas especies (Peña et al. 2014)

\section{Características botánicas del J. Neotropica}

Guambo (2018) argumenta que mediante la recopilación de la información documentada que realizó Toro y Roldán (2018) acerca de las características botánicas del J. neotropica en las que se identifica la altura que alcanza como árbol entre $15 \mathrm{~m}$ y $48 \mathrm{~m}$; raíz pivotante muy ramificada que supera los tres metros de profundidad; tronco cilíndrico, recto, que puede superar un metro de diámetro, cubierto por corteza externa fisurada color ceniciento y corteza interna fibrosa color crema. Posee una copa frondosa e irregular de hasta $10 \mathrm{~m}$ de ancho, con una gruesa ramificación 
de escaza médula, con lenticelas en adulto, ciertas veces con pubescencia rojiza, monopódicas y casi horizontales al fuste principal (Guambo, 2018).

Las hojas son compuestas alternas e imparipinadas agrupadas al final de las ramas; miden entre 20 $\mathrm{cm}$ y $60 \mathrm{~cm}$ de largo y $18 \mathrm{~cm}$ a $30 \mathrm{~cm}$ de ancho, que nacen a partir de una yema terminal escamosa, delgada y puntiaguda protegida por una bráctea vistosa y alargada. Contienen entre 7 y 19 foliolos dispuestos de manera opuesta en un raquis de pubescencia hirsuta; los foliolos frescos, cuando se maceran, desprenden un olor a melaza, miden entre $5 \mathrm{~cm}$ a $16 \mathrm{~cm}$ de largo y $2.5 \mathrm{~cm}$ a $8 \mathrm{~cm}$ de ancho y son sésiles.

El limbo presenta nervaduras reticuladas, con un haz rugoso de color verde oscuro; el envés es pubescente de color verde claro a blanquecino, de borde dentado a entero, tiene ápice agudo y base subcordada, dándole una apariencia o forma ovado lanceolados (Manning, 1978; Gómez y Toro 2007 citado en Guambo, 2018).

La polinización es principalmente anemófila representado en la Figura 1, dado por la gran producción de polen que producen los amentos, el período entre la germinación del polen en el estigma y la fecundación son 7 días. El fruto es una drupa globosa de exocarpio carnoso y fibroso, que al madurar libera el endocarpio leñoso que contiene el embrión, el cual es la parte comestible (Lemus et al.2010; Toro \& Roldán2018 citado en Guambo, 2018).

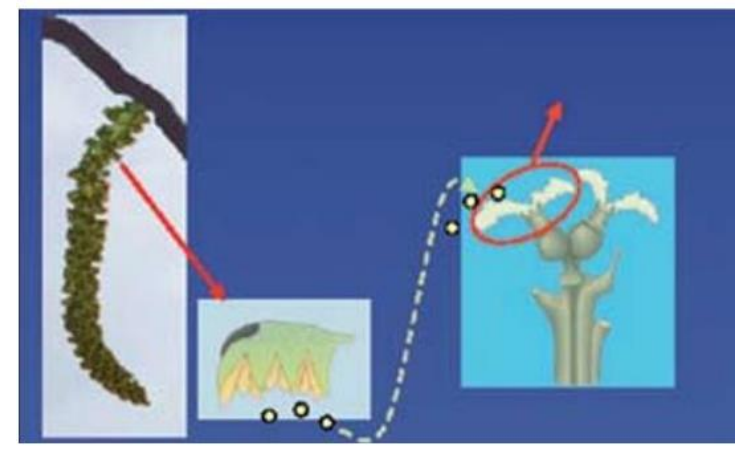

Figura 1. Polinización del Nogal

Fuente. (Lemus et al, 2010)

\section{Características fenológicas del J. neotropica}

Guambo (2018) menciona que a través de la fenología y productividad de los árboles semilleros se pueden elaborar calendarios o cronogramas que permitan una oportuna recolecta de frutos o 
semillas que se propagarán en vivero sin afectar el equilibrio del ecosistema, según lo analizado por Azas R. (2016).

De forma general Ospina et al. (2003) determinaron que la actividad reproductiva del J. neotropica es anual y dura ocho meses en todo el neotrópico, las fases fenológicas son influenciadas por factores ambientales y climáticos (altitud y precipitación).

Entre los meses de octubre a enero se presenta la floración, después de la caída de hojas, con una mayor duración floral para las espigas femeninas esto en base a varios estudios que describe Toro \& Roldán (2018), estas flores son polinizadas por abejas, atraídas por la gran cantidad de polen que producen las flores masculinas, quienes aportan a la polinización cruzada lejana, aunque la polinización más documentada para la especie es anemófila, esto se puede apreciar mediante la Figura 2.

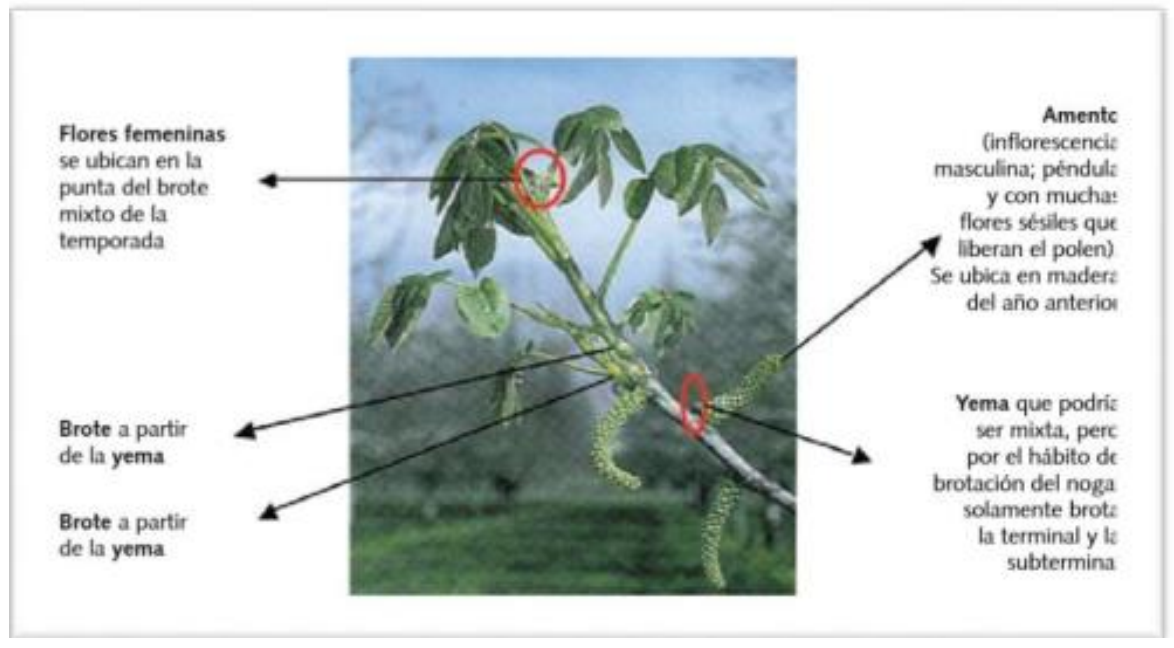

Figura 2 Órganos florales y vegetativos del nogal

Fuente: Lemus et al. (2010)

El proceso de fructificación se da de enero a septiembre, donde se encuentra alto aborto de frutos antes del periodo de maduración que comienza en el mes de junio, condición que puede ser entendida por procesos de hibridación es decir cruce reproductivo entre especies diferentes y que amerita de mayor investigación según lo expuesto por los autores Yamamoto y Barra (2003); Gómez \& Toro (2007).

Así también (Toro \& Roldán, 2018 citado en Guambo, 2019) recalcan que los frutos dispersados por animales y fuentes de agua alcanzan la madurez, determinando que frutos de seis meses de formación ya son aptos para iniciar su proceso de germinación y no antes, ya que estos frutos son 
climatéricos, esto es, que una vez caen del árbol ya no continúan con su proceso de maduración y desarrollo. En el Ecuador, la recolección de frutos se da entre los meses de agosto y octubre, cuando los frutos maduros inician su caída natural, recolectándolos directamente del suelo (Chusquillo, 2014). Según Lemus (2004) las semillas del nogal aparecen cuando se disgrega el mesocarpio del fruto, quedando la nuez o semilla con su cubierta característica como se muestra en la Figura 2.

La nuez tiene una fragancia suave y agradable. Chusquillo (2014 citado en Guambo, 2018) destaca que la semilla varia su tamaño de acuerdo al sitio de desarrollo 2.7 a $4.7 \mathrm{~cm}$ de largo, surcos amplios lisos y profundos en forma longitudinal, con embrión bien diferenciado y bastante aceitoso, con 2 a 4 lóbulos y dos cotiledones esculturados. Además, Toro y Roldán (2018) recomienda hacer la colecta en costales de fibra o cabuya para ser transportados al vivero para su desarrollo germinativo inmediato; no se recomienda almacenarlos por ser semilla recalcitrante (Guambo,2018).

\section{Requerimientos de fertilización del nogal j. neotropica}

El 90 a 92\% del peso seco total de un árbol es carbohidratos (componentes de la estructura del árbol). Según Daza (2018) en una sola planta se puede encontrar 16 elementos naturales, considerados primordialmente en el crecimiento de las plantas, los que se distribuyen de la siguiente manera: - Macronutrientes o macroelementos: carbono, hidrogeno, oxigeno, nitrógeno, fosforo, potasio, calcio, azufre y magnesio. - Micronutrientes o microelementos: hierro, cloro, manganeso, boro, cobre y molibdeno (Guambo, 2018).

\section{Macronutrientes}

Según la FAO (2002), los macronutrientes se necesitan en grandes cantidades, más aún cuando el suelo presenta déficit en uno o más de ellos, esto se debe a la extracción de nutrientes por los diversos cultivos durante años o a su vez en la utilización de rendimientos altos, las cuales son más demandantes en nutrientes que las variedades locales (Guambo, 2018). De acuerdo con Lemus et al. (2010) se puede apreciar en la Tabla 2 el déficit de $\mathrm{N}$ es muy común, así como el exceso suele ser poco común, se ha valorado comúnmente deficientes a K Y Mg (Guambo, 2018).

\section{Micronutrientes}

En su investigación Guambo (2018) indica que en contraste a los macronutrientes la FAO (2002) resalta que para el crecimiento correcto de las plantas los micronutrientes son demandados en 
cantidades mínimas, así mismo cuando no pueden ser provistos por el suelo se deben aplicar en cantidades muy pequeñas. Al 16 observar el cuadro de la presencia de déficit y exceso de micronutrientes en el nogal se puede establecer al Zn como el más común de todos (Lemus et al, 2010 citados en Guambo, 2018).

\section{Requerimientos nutricionales del Nogal}

Guambo (2018) reporta que en el primer año no se necesita aplicar la fertilización, más aún al realizar la plantación no se debe aplicar cerca de las raíces. Después de efectuada la plantación Loewe \& González (2001) recomiendan fertilizar con las cantidades como las que se muestran en la tabla uno.

Tabla 1. Recomendaciones de aplicación de nitrógeno y potasio en plantaciones

\begin{tabular}{cccc}
\hline $\begin{array}{c}\text { Año después de la } \\
\text { plantación }\end{array}$ & $\begin{array}{c}\text { Nitrógeno } \\
\text { (g/planta) }\end{array}$ & $\begin{array}{c}\text { Potasio } \\
\text { (g/planta) }\end{array}$ & $\begin{array}{c}\text { Radio de dispersión } \\
\text { alrededor del árbol }\end{array}$ \\
\hline 1 & 50 & 80 & 1 \\
2 & 100 & 160 & 1,5 \\
3 & 150 & 240 & 2 \\
4 & 200 & 320 & 2,5 \\
5 & 250 & 400 & 3 \\
6 & 450 & 800 & Bajo la copa \\
\hline
\end{tabular}

Fuente (Loewe \& González, 2001)

\section{Especificaciones de los nutrientes principales de las plantas.}

En su reporte, Guambo (2019) reporta las siguientes especificaciones de los nutrientes de las plantas:

La FAO (2002) cataloga NPK como los tres nutrientes primarios de todo cultivo, donde se establece a:

- Nitrógeno $(\mathrm{N})$ como el motor del crecimiento de la planta, también como componente esencial de las proteínas, se involucra en los procesos primordiales del desarrollo de las plantas y en el rendimiento. Un buen suministro de nitrógeno para la planta es importante también por la absorción de los otros nutrientes.

- Fósforo (P), tiene un papel importante en la transferencia de energía, porque es esencial para la fotosíntesis y otros procesos químico-fisiológicos. Es imprescindible para la 
diferenciación de las células y el desarrollo de los tejidos, que establecen los puntos de crecimiento de la planta. El fósforo es deficiente en la mayoría de los suelos naturales o agrícolas o dónde la fijación limita su disponibilidad.

- Potasio (K), tiene varias funciones, entre las cuales resaltan; la activación de más 60 enzimas, por lo que toma un vital papel en la síntesis de carbohidratos y proteínas. El régimen hídrico de la planta es mejorado por el $\mathrm{K}$ e incrementa su tolerancia a la sequía, heladas y salinidad. Las plantas bastante dotadas con K tienen escasas probabilidades de sufrir enfermedades.

\section{Metodología}

La investigación se realizó en el vivero o umbráculo forestal de la Facultad de Recursos Naturales perteneciente a la Escuela Superior Politécnica de Chimborazo ubicada en la parroquia Lizarzaburu, cantón Riobamba, provincia de Chimborazo, a una latitud de $01^{\circ} 30^{\prime} \mathrm{S}$, longitud de 7840`W 586 y altitud de 2820 msnm, en la sierra centro de la República del Ecuador (Guambo, 2018).

Se utilizó el diseño de Bloques Completos al Azar (BCA) desbalanceado, Se estudiaron 56 tratamientos en 262 observaciones distribuidas entre los tratamientos, con el objetivo de demostrar los efectos de disoluciones nutritivas en el desarrollo de J. neotrópica, las mediciones semanales se realizaron desde el 29 de junio de 2017 hasta 31 de agosto de 2017 con intervalos que permitieron ejecutar 7 mediciones en 65 días de investigación, y con esto se generó la base de datos para el análisis (Guambo, 2018).

\section{Implementación del experimento}

Guambo (2018) desarrolló la investigación en una sola etapa experimental la cual cuenta de distintas tareas para alcanzar los objetivos propuestos.

1. Instalación del experimento

2. Regar con $200 \mathrm{ml}$ de disoluciones nutritivas cada 72 horas

3. Toma de datos de las características morfológicas las variables mencionadas de acuerdo con el cronograma.

4. Sistematización de los datos de las características morfológicas

5. Determinación de estadísticos de dispersión y tendencia central 
Tabla 2. Procedencia de las especies de J neotropica (Factor A)

\begin{tabular}{lcc}
\hline \multicolumn{1}{c}{ Procedencia } & Código & Repeticiones \\
\hline Chambo & P1 & 43 \\
Guamote & P2 & 36 \\
Riobamba & P3 & 38 \\
Mix Chimborazo & P4 & 37 \\
Ambato & P5 & 38 \\
Guaranda & P6 & 37 \\
Mix Tungurahua & P7 & 33 \\
\hline
\end{tabular}

Fuente: levantamiento en campo, 2017.

Realizado por: Guambo, Verónica. 2018

\section{Disoluciones Nutritivas (Factor B)}

Se evaluaron siete disoluciones nutritivas con distinta concentración de (N-P-K) Tabla 3 y la octava fue el testigo (Guambo, 2018).

Tabla 3. Concentración de las disoluciones nutritivas a utilizarse en la investigación

\begin{tabular}{llllll}
\hline Disoluciones & $\mathrm{N}(\mathrm{ppm})$ & $\mathrm{P}(\mathrm{ppm})$ & $\mathrm{K}(\mathrm{ppm})$ & Codificación & Repeticiones \\
\hline 1 & 00.00 & 100.00 & 100.00 & $\mathrm{~T} 1$ & 35 \\
2 & 25.00 & 100.00 & 100.00 & $\mathrm{~T} 2$ & 32 \\
3 & 50.00 & 100.00 & 100.00 & $\mathrm{~T} 3$ & 30 \\
4 & 50.00 & 0.00 & 100.00 & $\mathrm{~T} 4$ & 30 \\
5 & 50.00 & 50.00 & 100.00 & $\mathrm{~T} 5$ & 32 \\
6 & 50.00 & 100.00 & 0.00 & $\mathrm{~T} 6$ & 33 \\
7 & 50.00 & 100.00 & 50.00 & $\mathrm{~T} 7$ & 34 \\
8 & 0.00 & 0.00 & 0.00 & $\mathrm{~T} 8$ & 36 \\
\hline TOTAL & & & & & 262 \\
\hline
\end{tabular}

Fuente: *ppm. Refiere a mg/L

Realizado por: Guambo, Verónica. 2018

\section{Análisis e interpretación de datos}

Guambo (2018) analiza los datos y reporta que la información muestra las medias de los tratamientos respecto a los registros de altura de las plántulas en experimentación, se puede observar la media tanto para las procedencias en estudio, así como también para las soluciones nutritivas que se aplicaron en cada planta; los parámetros de dispersión y tendencia central de toda la población son considerados para la aplicación del DBCA con las procedencias como Bloque. Nótese las diferencias estadísticas en las plántulas según su procedencia al inicio de la 
investigación; en cambio modelo DBCA no permite aparecer diferencias en la asignación de los tratamientos.

Tabla 4. Altura de plántulas $(\mathrm{cm})$ de J. neotropica inicio de investigación

\begin{tabular}{llllllll|lll}
\hline & P1 & P2 & P3 & P4 & P5 & P6 & P7 & Media & DT & CV \\
& a & ab & b & ab & ab & ab & b & & & \\
\hline T1 & 26,35 & 21,18 & 16,00 & 16,73 & 16,66 & 19,93 & 16,90 & 19,11 & 3,46 & 18,09 \\
T2 & 27,44 & 20,90 & 21,75 & 26,38 & 21,56 & 22,13 & 15,67 & 22,26 & 3,58 & 16,09 \\
T3 & 27,27 & 15,60 & 20,08 & 21,44 & 21,88 & 21,76 & 18,68 & 20,96 & 3,30 & 15,75 \\
T4 & 24,24 & 18,55 & 18,43 & 20,65 & 21,00 & 19,10 & 14,88 & 19,55 & 2,66 & 13,61 \\
T5 & 22,88 & 19,67 & 16,19 & 17,47 & 19,80 & 18,40 & 19,97 & 19,20 & 1,98 & 10,31 \\
T6 & 21,77 & 22,04 & 9,90 & 20,74 & 17,30 & 17,72 & 16,50 & 18,00 & 3,90 & 21,67 \\
T7 & 22,60 & 21,55 & 18,06 & 15,63 & 20,23 & 18,30 & 17,71 & 19,15 & 2,24 & 11,71 \\
T8 & 25,63 & 24,83 & 20,30 & 21,43 & 14,67 & 21,86 & 13,30 & 20,29 & 4,36 & 21,51 \\
Media & 24,77 & 20,54 & 17,59 & 20,06 & 19,14 & 19,90 & 16,70 & 19,81 & & \\
DT & 2,07 & 2,53 & 3,45 & 3,20 & 2,45 & 1,68 & 1,99 & & $\mathbf{3 , 5 0}$ & \\
CV & 8,34 & 12,33 & 19,63 & 15,96 & 12,79 & 8,42 & 11,91 & & & $\mathbf{1 7 , 6 8}$ \\
\hline
\end{tabular}

Fuente: Letras iguales indican semejanza estadística.

Realizado por: Guambo, Verónica. 2018

La desviación típica (DT) general fue de $\pm 3,50$ respecto de la media poblacional que al inicio de investigación fue de 19,81 cm, el trabajo experimental se inició con un coeficiente de variación (CV) de 17,18\%. Mediante un ANOVA de efectos principales de cada factor del experimento, se descartó la hipótesis nula sobre igualdad en la respuesta de crecimiento de las plantas de J. neotropica, frente a la aplicación de siete soluciones nutritivas y un tratamiento control sin aporte nutricional (Guambo, 2018). La diferencia encontrada suele deberse a la 33 disponibilidad inmediata para la planta de estos nutrientes en el sustrato o suelo sumado a los aportes realizados con las disoluciones nutritivas (Loewe \& González, 2001).

Tabla 5.Análisis de varianza efecto de disoluciones nutritivas en incremento de altura

\begin{tabular}{llllll}
\hline Origen & SC & GL & CM & F & Sig. \\
\hline Modelo & $620,097^{\mathrm{a}}$ & 14 & 44,293 & 273,365 &, 000 \\
Procedencia & 2,925 & 6 &, 488 & 3,009 &, 007 \\
Disolución & 168,675 & 7 & 24,096 & 148,718 &, 000 \\
Error & 40,183 & 248 &, 162 & & \\
Total & 660,280 & 262 & & & \\
Total corregida & 213,853 & 261 & & \\
\hline a. R cuadrado $=, 939$ (R cuadrado corregida $=, 936)$ & \\
\hline
\end{tabular}

Realizado por: Guambo, Verónica. 2018 
La Tabla 5 demuestra que en el estudio realizado la utilización de diferentes disoluciones tiene efecto sobre el desarrollo de J. neotropica, se encontraron diferencias estadísticas altamente significativas entre los tratamientos y respecto del control (T8). Además, las diferencias entre los orígenes de las plántulas fueron altamente significativas, el sustrato utilizado en el experimento permite realizar un símil con lo publicado por Cabascango (2011), donde, con la utilización de arena y tierra de páramo se pudo demostrar que la plántula recibe bien la tierra negra para producir mayor calidad de raíces y en consecuencia mejores alturas de la planta en su desarrollo inicial, así también, Favela et al. (2006), exponen la importancia de las demandas nutritivas de la semilla una vez que esta emerge; mientras mayor sea la eficiencia de alimentación vegetal los parámetros de crecimiento serán afectados de manera favorable. Bellote \& Ferreira (1995) en relación a la fertilización observaron el efecto positivo de la aplicación de fósforo (P) si se considera como una actividad regular en plantaciones pues según avanza la edad de la planta la demanda se incrementa, pero la oferta no; por esto, fertilizar proporciona mayor absorción y, en consecuencia, mayor crecimiento de los árboles. Uscola et al. (2009) interpretaron que la fertilización nitrogenada produce cambios en las especies forestales expresados como una reducción del tamaño de la raíz, esto debido a que las plantas priorizan el desarrollo radicular sobre el desarrollo aéreo, pero también depende del tipo de nitrógeno disponible pues el desarrollo aéreo se da mejor en presencia de NO3 - siendo más eficiente para desarrollar raíces fuertes el $\mathrm{NH}_{4}+(\mathrm{Guambo}, 2018)$.

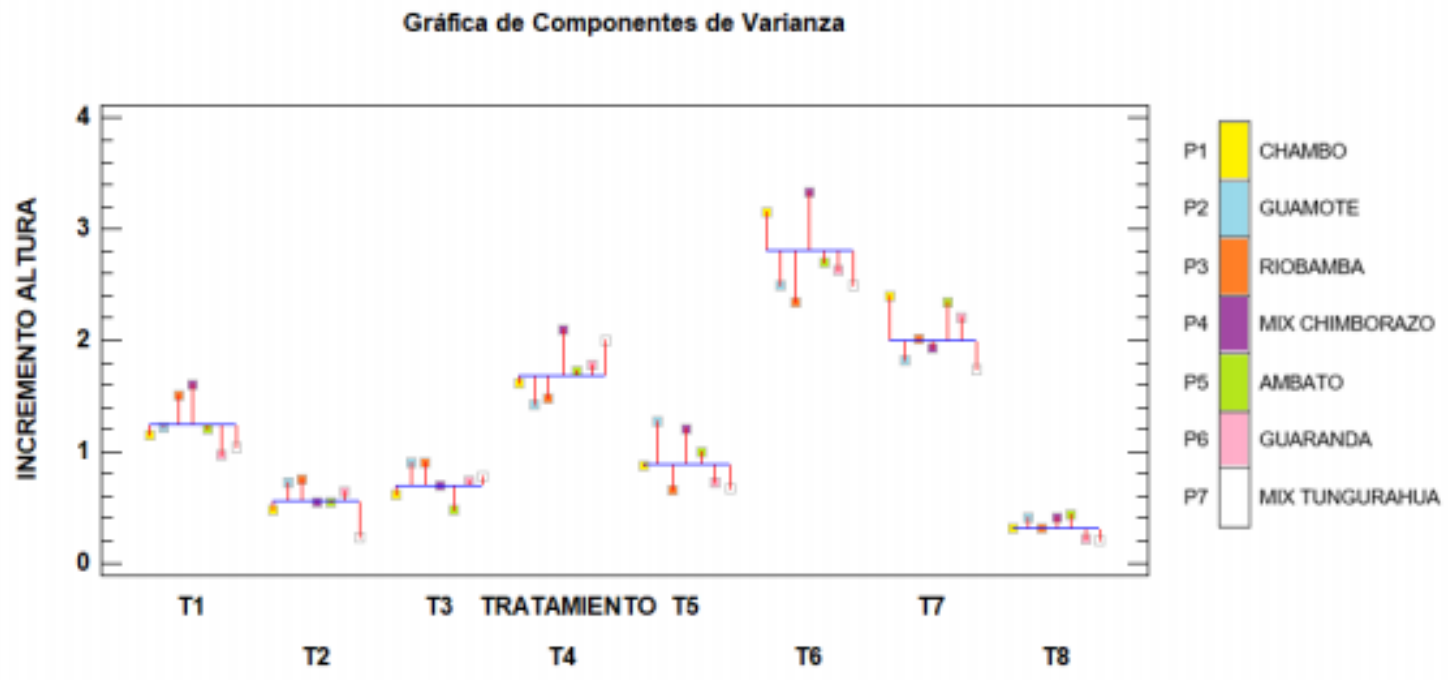

Figura 1. Efecto de disoluciones nutritivas en altura $(\mathrm{cm}) \mathrm{J}$ neotropica de diferentes 
La ilustración 1, permite observar las diferencias estadísticas encontradas por el ANOVA, en centímetros se evidenció que los mejores incrementos respecto de la altura fueron los alcanzados por T6 en P4, se encontró congruencia entre los valores alcanzados por las distintas disoluciones y el desarrollo de P4 fue superior a las demás medidas principalmente respecto de P7 T8 que tuvo el récord más bajo de incremento de altura en la duración del experimento. Autores como Valverde (2016); y Azas (2016), describen al Nogal como una especie muy exigente al momento de su plantación por las demandas de Nitrógeno, Fosforo, Potasio y recomiendan antes de plantar realizar un abonado de fondo y así determinar la carencia de nutrientes del suelo por lo que se sugiere que cuando el suelo sea ácido en extremo se añadan dosis pequeñas de cal para evitar el bloqueo de otros elementos en función del pH y textura de suelo (Guambo, 2018).

Favela et al. (2006) exponen sobre la calidad de las Soluciones nutritivas (SN) y la importancia de las dosis, la conductividad, la relación entre cationes y aniones, cantidad de amonio, oxígeno disuelto y temperatura; además, este autor hace recomendaciones para el uso de SN en la investigación científica. Expone la cantidad de los macronutrientes (CHONPS) cercana al 98\% dejando la diferencia para las cenizas. En orden de abundancia después del carbono, el hidrógeno, el oxígeno y el nitrógeno se encuentra en las plantas en forma inorgánica y orgánica principalmente dentro de las proteínas los ácidos nucleicos y los aminoácidos su concentración foliar oscila entre 1,5 a $6 \%$ de la materia seca en los cultivos (Guambo, 2018).

Favela et al. (2006 citado en (Guambo, 2018), remarcaron la importancia del fósforo (P) al formar parte de las enzimas, proteínas, adenosina trifosfato responsable de la transferencia de energía y la genética por medio del ADN y ARN, sus concentraciones respecto de la materia seca pueden alcanzar el 1\%; en cambio, el potasio $(\mathrm{K})$ es responsable del estado del agua en planta como activador en varios procesos que permiten la turgencia en el nivel celular y la translocación de carbohidratos recién elaborados en la concentración de materia seca se lo puede hallar hasta en $5 \%$. Cabascango (2011) a los 30 días reporta alturas de hasta 5,47 cm muy por debajo de los 20,17 de este estudio, pero a los 75 días los datos reportados por este autor llegan a 22, $26 \mathrm{~cm}$ que supera numéricamente los 21,10 cm de este estudio a los 95 días de edad de la planta. 
Tabla 6. Separación de medias incremento de altura $(\mathrm{cm})$ de J. neotropica

\begin{tabular}{lllllllll}
\hline DISOLUCIÓN & N & \multicolumn{2}{c}{ Subconjunto } & & & & \\
& & 1 & 2 & 3 & 4 & 5 & 6 & 7 \\
\hline T8 & 32 &, 3156 & & & & & & \\
T2 & 32 &, 5594 &, 5594 & & & & & \\
T3 & 32 & &, 7000 &, 7000 & & & & \\
T5 & 32 & & &, 8906 & & & & \\
T1 & 32 & & & & 1,2406 & & & \\
T4 & 33 & & & & & 1,6788 & & \\
T7 & 32 & & & & & & 1,9938 & \\
T6 & 37 & & & & & & & 2,8162 \\
Sig. & &, 223 &, 851 &, 542 & 1,000 & 1,000 & 1,000 & 1,000 \\
\hline
\end{tabular}

Realizado por: Guambo, Verónica. 2018

La tabla 6, muestra las diferencias a un nivel de confianza 0,05 según la prueba de Tukey, si se observa las disoluciones es evidente un mayor desarrollo de las plántulas alimentadas con la disolución nutritiva T6 (N50ppm. P100 ppm. K0 ppm.) obsérvese que se forman siete grupos en los que se diferencian los efectos de los nutrientes aplicados, sin embargo no se encontraron diferencias significativas entre T8 (testigo sin nutrientes adicionados) y T2( N25 ppm, P100 ppm, K100 ppm), así también T2 y T3 y esta respecto de T5, al ser T8 el testigo se puede afirmar que el uso de T2 es ineficiente y muestra un efecto favorable respecto de su contraparte es decir este grupo de disoluciones, de las que se definió como beneficiosa a T5, y se puede observar tanto la figura 9 como en la tabla 9 la superioridad de T6 que redondea 2,82 cm de crecimiento en los 65 días de evaluación (Guambo, 2018).

\section{Conclusiones}

- Como parte importante de esta investigación se evaluó la fertilización de la especie de J. neotropica con disoluciones nutritivas para una eficiente producción de plantines en vivero, con lo cual se determinaron las soluciones nutritivas que se ajustan a los requerimientos de esta especie que permitió obtener plantas de calidad que aseguren la supervivencia, el crecimiento y una satisfactoria aclimatación en el campo.

- Las proporciones de N P K más efectivas se encuentran entre T7 (N50 ppm, P100 ppm, K 50 ppm), y T6 (N50 ppm, P100 ppm, K 0ppm), pues se demostró que por cada unidad de 
Nitrógeno las plantas de Juglans neotrópica reciben de manera positiva dos unidades de Fósforo, por otro lado, los mejores resultados se encuentran en proporciones nulas de Potasio.

- Al utilizar la técnica estadística clúster se obtuvieron tres grupos de muestras sobre la base de la respuesta morfológica de las plantas de J. neotrópica, y con el estadístico Chi cuadrado de Pearson se encontró una asociación entre los grupos del clúster y las procedencias, por lo que, se definieron argumentos para sustentar una duda razonable y se afirma que dentro de las semillas utilizadas pueden existir subvariedades que han influenciado grandemente en los resultados.

\section{Referencias Bibliográficas}

Azas, R. (2016). Evaluación del efecto de los tratamientos pregerminativos en semillas de nogal (Juglans neotrópica Diels) en el recinto Pumin provincia de Bolívar”. Tesis de Pregrado. Santo Domingo de los Tsáchilas, Ecuador: Universidad de las Fuerzas Armadas ESPE.

Basave, E., López, M., Cetina, V., Aldrete, A., \& Almaraz, J. (2014). Prácticas culturales en vivero que influyen en la calidad de planta de Enterolobium cyclocarpum. Bosque (Valdivia).

Cabascango, M. (2011). Evaluación de cuatro tipos de sustratos y tres niveles de humus en la obtención de plántulas de nogal (juglans neotrópica) en la zona de Otavalo, Provincia de Imbabura. Tesis de pregrado. Universidad Técnica De Babahoyo.

Favela, E., Preciado, P., \& Benavidez, A. (2006). Manual para la preparación de soluciones nutritivas. Torreón, Coahuila: Universidad Autónoma Agraria Antonio Narro, ISBN 968844-051-5.

Guambo, V. (2018). "Efectos de disoluciones nutritivas de nitrógeno, fósforo y potasio (n-p-k) sobre el crecimiento de plántulas de Juglans neotropica Diels”.

Lemus, G., Ibacache, A., Pinilla, B., Riveros, F., Sellés, G., Ferreira, R., . . . Vial, J. (agosto de 2010). Producción de nueces de Nogal. Manuales FIA de Apoyo a la Formación de Recursos Humanos para la Innovación Agraria. Santiago, Chile. 
Jara, J. C. (2015). "La deforestación de los bosques protectores como un atentado al Derecho al Buen Vivir en la Legislación Ecuatoriana”. Quito.

Ramos, V. A., \& Arias, M. (2016). Análisis espacial de cuencas hidrográficas como soporte a la gestión territorial y ambiental. caso de estudio: cuenca del río Arenillas. Obtenido de Tesis de Maestría en Impactos Ambientales, Universidad de Guayaquil: http://repositorio.ug.edu.ec/bitstream/redug/11977/1/TESIS\%20\%20VER\%C3\%93 NICA\%20RAMOS\%20FINAL.pdf

Salto, C., Harrand, L., Oberschelp, G., \& Ewens, M. (2016). Crecimiento de plantines de Prosopis alba en diferentes sustratos, contenedores y condiciones de vivero. Bosque, vol. 37, núm. $3,527-537$.

Toro, E., \& Roldán, I. C. (2018). Estado del arte, propagación y conservación de Juglans neotropica Diels., en zonas andinas. Madera bosques vol.24 no.1 Xalapa abr. 2018, http://dx.doi.org/10.21829/myb.2018.2411560.

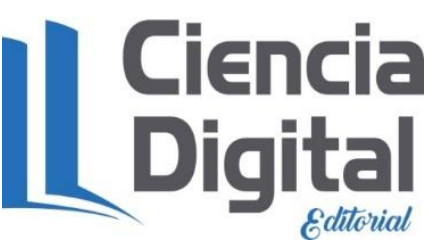




\section{PARA CITAR EL ARTÍCULO INDEXADO.}

Guambo Tapia, V. S., Valenzuela Erazo, D. A., Saeteros Hernández, A. M., \& Noboa Silva, V. F. (2020). Efectos de disoluciones nutritivas de nitrógeno, fósforo y potasio (N-P-K) sobre el crecimiento de plántulas de Juglans Neotropica Diels. Ciencia Digital, 4(1), 236-252. https://doi.org/10.33262/cienciadigital.v4i1.1097

\section{¿Ciencia}

El artículo que se publica es de exclusiva responsabilidad de los autores y no necesariamente reflejan el pensamiento de la Revista Ciencia Digital.

El artículo queda en propiedad de la revista y, por tanto, su publicación parcial y/o total en otro medio tiene que ser autorizado por el director de la Revista Ciencia Digital.
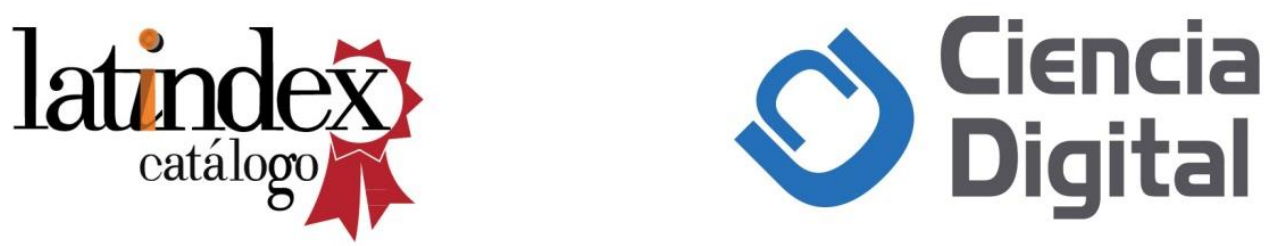